\title{
Challenges in Control and Autonomy of Unmanned Aerial-Aquatic Vehicles
}

\author{
André Farinha ${ }^{1}$, Julien di Tria ${ }^{1}$, Raphael Zufferey ${ }^{1,2}$, Sophie F. Armanini ${ }^{1,3}$ and Mirko Kovac ${ }^{1,4, *}$
}

\begin{abstract}
Autonomous aquatic vehicles capable of flight can deploy more rapidly, access remote or constricted areas, overfly obstacles and transition easily between distinct bodies of water. This new class of vehicles can be referred as Unmanned Aerial-Aquatic Vehicles (UAAVs), and is capable of reaching distant locations rapidly, conducting measurements and returning to base. This greatly improves upon current solutions, which often involve integrating different types of vehicles (e.g. vessels releasing underwater vehicles), or rely on manpower (e.g. sensors dropped manually from ships). Thanks to recent research efforts, UAAVs are becoming more sophisticated and robust. Nonetheless numerous challenges remain to be addressed, and particularly dedicated control and sensing solutions are still scarce. This paper discusses challenges and opportunities in UAAV control, sensing and actuation. Following a brief overview of the state of the art, we elaborate on the requirements and challenges for the main types of robots and missions proposed in the literature to date, and highlight existing solutions where available. The concise but wide-ranging overview provided will constitute a useful starting point for researchers undertaking UAAV control work.
\end{abstract}

\section{INTRODUCTION}

Unmanned Aerial-Aquatic Vehicles (UAAVs) are unmanned vehicles that inherit traits from both Unmanned Aerial Vehicles (UAVs) and Autonomous Underwater Vehicles (AUVs), and are thus able to move through both air and water. Especially at small sizes, such robots are highly promising to enable new types of missions in aquatic environments, as well as to simplify or improve existing procedures. Flight enables UAAVs to cover distances rapidly, avoid obstacles and move between separate bodies of water, while being able to carry out a variety of tasks that involve water interaction, such as water sampling, marine investigation, monitoring of fragile environments (such as coral reefs), or industrial maintenance work (for instance around offshore platforms). State of the art designs achieve this particular type of multimodal locomotion through adaptable physical morphology [1] and/or control strategy [2] adaptation, sometimes inspired by nature [3]. Literature on UAAVs is extensive [4] and covers vehicles ranging from the micro-scale to several meters wingspan, as well as a variety of flying vehicle categories, i.e. fixed-wing, rotorcraft, ornithopters and jet-thrusters, as shown in Table I.

Despite a considerable body of research on such robots over the past few years [5], [6], UAAVs remain challenging to develop,

\footnotetext{
${ }^{1}$ Aerial Robotics Laboratory, Imperial College London, UK

${ }^{2}$ Robotics, Vision and Control Group, University of Seville, Spain.

${ }^{3}$ Faculty of Aerospace and Geodesy, Technical University of Munich, Germany.

${ }^{4}$ Materials and Technology Centre of Robotics, Swiss Federal Laboratories for Materials Science and Technology, Switzerland.

Authors contributed equally.

* Corresponding author: Mirko Kovac, e-mail: m.kovac@imperial.ac.uk
}

and fully functioning autonomous and robust prototypes are still scarce. To date, the vast majority of existing studies have focused on the design aspect, i.e. achieving locomotion in both mediums and transitioning between the two [7]-[11]. Far less work has flown into the control and autonomy side, with most prototypes remaining at an early demonstration stage.

In this short paper we discuss the central challenges involved in control and autonomous operation of UAAVs. Starting from a succinct overview of recent developments in the literature, we highlight gaps in the current body of work, placing particular emphasis on distinguishing between the specific challenges pertaining to the different UAAV categories proposed so far.

\section{MISSION PROFILES}

UAAVs can perform a wide range of different missions and are suited for a variety of different applications. Example mission profiles compatible with selected UAAVS listed in Table I are defined below and illustrated in Fig. 1.

A Fly to target $\rightarrow$ land on water $\rightarrow$ perform long duration mission $\rightarrow$ take-off from water $\rightarrow$ fly back to base or on to next target. [fixed-wing or multirotor landing on water, e.g. SailMAV by Zufferey et al. [12]]. This profile suits long (in range and in duration) survey missions, like sea-bed mapping, or shore monitoring.

B Fly to target $\rightarrow$ dive into water $\rightarrow$ perform underwater task $\rightarrow$ transition back to flight $\rightarrow$ fly back to base or on to next target. [fixed-wing or multirotor able to move underwater, e.g. AquaMAV by Siddall et al. [13] or Looncopter by Alzubi et al. [9]]. Shorter and faster missions like pin-point water-sampling, emergency deployment are covered here.

C Fly to target $\rightarrow$ land on water $\rightarrow$ release tethered sensing unit underwater $\rightarrow$ retrieve released unit $\rightarrow$ take off from water $\rightarrow$ fly back to base. [multi-part robot, e.g. MEDUSA by Debruyn et al. [14]]. When short range, precise underwater locomotion and hard-to-access location are required, for example in coral-reef monitoring.

D Aquatic based launch $\rightarrow$ ( passively acquire data $\rightarrow$ jumpglide to new location ) repeat $\rightarrow$ aquatic recovery. [e.g. the robot described by Zufferey et al. [15]]. This open the doors for overcoming a lot of small obstacles, for example jumping from ponds to ponds, and perform chlorophyll measurements.

In order to illustrate the challenges of each mission type more effectively, we will discuss specific robots as demonstrative examples throughout the paper. 
Table I: Review of UAAV literature.

\begin{tabular}{|c|c|c|c|}
\hline Robot name & Aircraft type & Water navigation & Key features \\
\hline SUWAVE [16], [17] & Fixed-wing, rotorcraft & Water surface & VTOL \\
\hline MEDUSA [14] & Quadcopter, jet-thruster & Underwater & $\begin{array}{l}\text { Two agents, quadcopter, } \\
\text { underwater pod }\end{array}$ \\
\hline SailMAV [12] & Fixed-wing, sailing & Water surface & Morphing wing \\
\hline AquaMAV [4], [7] & Fixed-wing, jet-thruster & Underwater & $\begin{array}{l}\text { Morphing wing, plunge diving, } \\
\text { wet launch }\end{array}$ \\
\hline Aquatic jump-glider [15] & Fixed-wing, jet-thruster & Air glide, water surface & Multiple jumps, wet launch \\
\hline ACAT [18], Flying Fish Solar-Powered [19] & Fixed-wing, seaplane & Water surface & Land on Water, running takeoff \\
\hline $\begin{array}{l}\text { Kollmorgen Sea Sentry 2009, Darpa Cormorant } 2005 \\
\text { and XFC Sea Robin } 2013 \text { [20] }\end{array}$ & Fixed-wing, submarine launched & Underwater & dry launch, morphing wings \\
\hline Beihang Flying Fish [21] & Fixed-wing, rotorcraft, jet-thruster & Underwater, surface & Morphing wings, wet launch, ballast \\
\hline MIT flying fish [22] & Fish swimming (undulation) & Air glide, underwater & Underwater speed $5 \mathrm{~m} / \mathrm{s}$ \\
\hline Quad-H2o, Mariner, HUAUV [23], Loon Copter [9] & Quadrotor & Underwater & Dual-propeller system \\
\hline Flying jellyfish [24] & Ornithopters & Underwater, surface & Self stabilizing without sensors, \\
\hline RoboBee [25] & Ornithopters & Underwater & Leaping out of water, micro scale \\
\hline
\end{tabular}

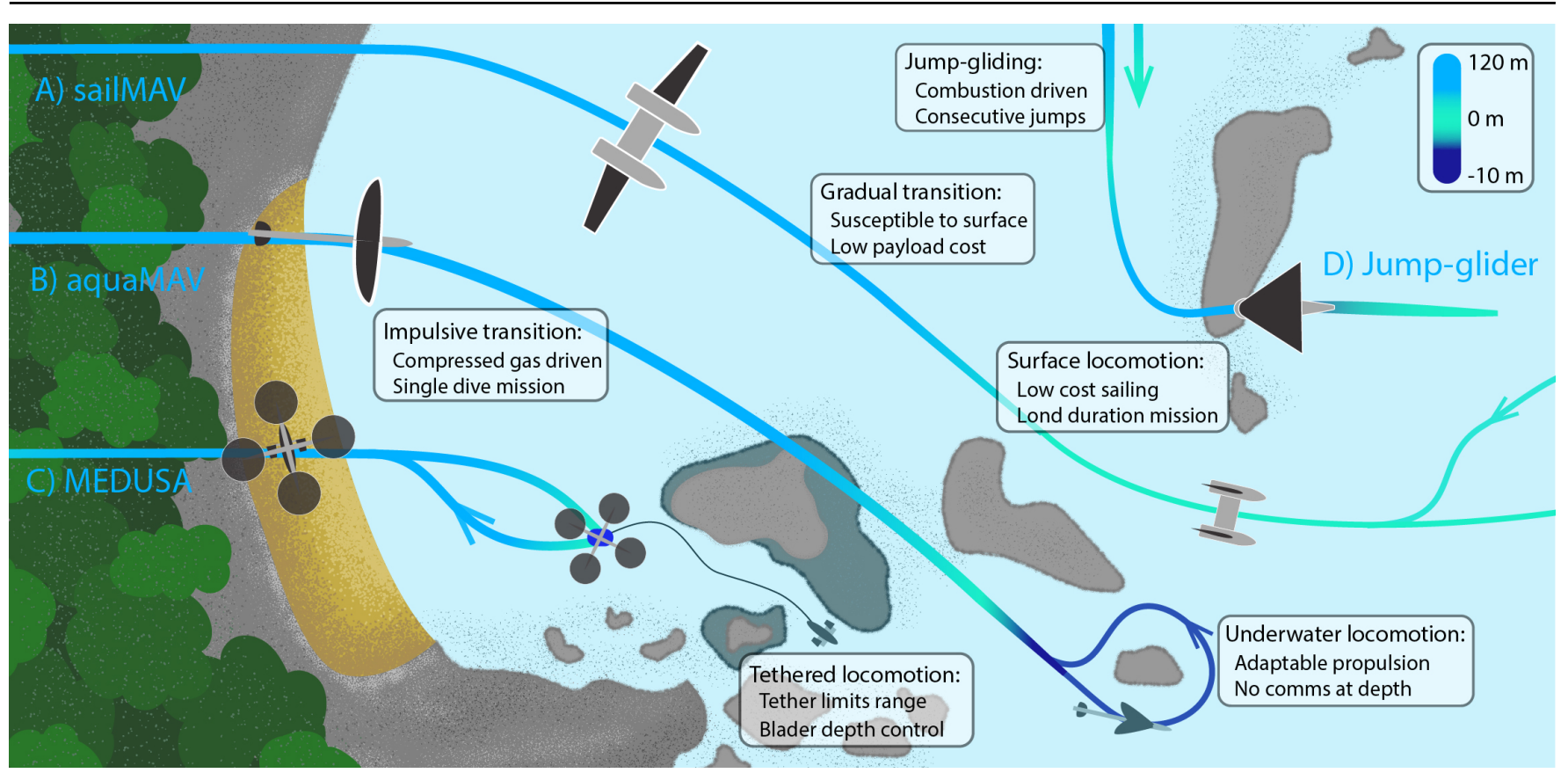

Figure 1: Example mission profiles envisioned for four UAAVs.

\section{CONTROL AND AUTONOMY IN AERIAL-AQUATIC ROBOTS}

\section{A. Challenges in aerial-aquatic control}

Automatic operation and control of UAAVs involves a number of additional considerations compared to purely aerial or aquatic robots, due to having to consider different modes of locomotion, and the transitions between those. During its different modes of operation, a UAAV is in many ways analogous to a fully aerial or fully aquatic vehicle, and therefore conventional control laws can be applied [9], [14]. However, the design and actuation particularities involved in many UAAVs may introduce unusual constraints (cf. Sec. IV). In the SailMAV sailing-flying robot [12], for example, roll control in flight needs to be handled by rotating the full wings, as this type of actuation is required for sailing. The aforementioned robot is also an example of a UAAV involving unusual modes of locomotion (in this case sailing), which requires additional control and sensing components. Thus for this particular robot an autonomous sailing control system was proposed, which orients the sails appropriately based on a novel wind sensor (see also Sec. IV).
In addition to the above points, the different requirements in the air and on/under the water may call for different control gains or even different control laws, as well as smooth switching between these. This in turn requires the vehicle to autonomously determine what state it is in [2], and to smoothly switch between controllers at the right moment to avoid instabilities.

The most challenging steps within the air-water-air cycle, however, are the transitions from water to air and from air to water. Firstly, the physical differences between the two media (density, viscosity) imply that the aerodynamic surfaces are mismatched (propellers and wings). This aspect can be mitigated through the use of adaptive morphology [1] or change in propeller speeds [26]. In the case of the propellers, an alternative solution may also be to include several propellers in the design, with each being optimised for operation in a different media [23]. Secondly, the water surface state outside of controlled lab environments is always chaotic and unpredictable, involving both waves and currents. The small scale of Micro Aerial Vehicles (MAVs), typically below $1 \mathrm{~m}$ length, 
further magnifies the impact of the water surface on the vehicles.

In order to reliably transition between media, UAAVs need enhanced mechanical intelligence to operate during the transition, and control methods that maintain stability before, during and after transition [27]. Broadly speaking, transitions methods can be either impulsive or gradual. These two modalities have different requirements and implications summed up in Table II.

Table II: Transition modality

\begin{tabular}{|c|c|c|}
\hline & Impulsive & Gradual \\
\hline Escape & $\begin{array}{l}\text { High impulse propulsion from } \\
\text { energy stored as a chemical, } \\
\text { elastic element or compressed } \\
\text { gas }\end{array}$ & $\begin{array}{l}\text { Low impulse propulsion from } \\
\text { aerodynamic surfaces }\end{array}$ \\
\hline Entry & $\begin{array}{l}\text { Subject to high impact forces } \\
\text { due to high speed }\end{array}$ & $\begin{array}{l}\text { Slow approach, subject to } \\
\text { sea-state }\end{array}$ \\
\hline \multirow[t]{3}{*}{ Robots } & AquaMAV [13] & SailMAV [12] \\
\hline & Aquatic jump-glider [15] & MEDUSA [14] \\
\hline & Robobee [28] & SUWAVE [16], [17] \\
\hline Control & $\begin{array}{l}\text { High rapidity, accurate models } \\
\text { and physical intelligence are } \\
\text { more effective than active } \\
\text { control }\end{array}$ & $\begin{array}{l}\text { Low rapidity, sensors can } \\
\text { acquire information on the } \\
\text { environment and allow oper- } \\
\text { ations in tougher conditions }\end{array}$ \\
\hline Cost & $\begin{array}{l}\text { Additional on-board power } \\
\text { storage }\end{array}$ & Extra sensors and actuators \\
\hline Limitation & Finite number of launches & $\begin{array}{l}\text { Sea state may hinder landing } \\
\text { and take-off }\end{array}$ \\
\hline
\end{tabular}

The dynamics of impulsive and gradual transition are considerably different. While the first lasts a fraction of a second and effects such as added water mass, play an important role, the second covers larger time scales where ground effect and the influence of sea state are substantial. The complexity of the aforementioned factors also implies that even though many analytical tools have been shown in the literature, empirical models and exhaustive experimentation still hold an important place in UAAV development.

\section{B. Impulsive transition modeling and control}

Jet propulsion has received considerable attention in the past. Generally, the modelling process is separated into external dynamics and internal thermodynamics. The internal thermodynamics can be modelled as an adiabatic expansion and the produced thrust inferred from Bernoulli's equation as in [7]. The one-way coupling of internal to external dynamics is widely covered in the literature due to the significant mass of water stored in water thrusters. The reverse coupling consists in the effect of inertial forces on the stored water, and is covered in [15].

One of the crucial effects to be taken into account during transition is the added mass, a general term which concerns inertial forces imposed on an immersed body, due to acceleration of either the fluid or the body itself. Such an effect is normally difficult to measure experimentally as it occurs alongside other processes; likewise, analytical approaches tend to not take into account the somewhat complex geometries of UAAVs.

Another similar though distinct effect occurring solely during water exit is water entrapment. This temporarily increases the vehicle's payload, and can occur in the vehicle's cavities such as the wings, or on the vehicle's surface due to wetting. The former type occurs when large floodable cavities are used for buoyancy management, and can be estimated if adequate ventilation is provided, so that air can replace the draining cavity [29]. The latter type is inevitable and often neglected, though considerable improvements can be obtained by reducing the surface energy of UAAVs' surfaces using methods such as surface etching, laser engraving and nanoparticle deposition.

Due to their inherent rapidity, impulsive transitions typically do not involve active control until the vehicle is clear of the water. During this phase, it is difficult to influence the system in a reliable and accurate way, hence it is important to ensure that the initial conditions before take-off (e.g. orientation) are set correctly [30]. To ensure this, it is in turn valuable to have accurate models of the entire process (cf. previous paragraph), allowing for the impulsive take-off trajectories to be predicted. Once the vehicle is outside the water, on the other hand, active control is useful to ensure a smooth and stable transition from jetting to flight. In UAAVs involving structural morphing [4], the unfolding of the wings poses an additional challenge and must be accurately timed to ensure uninterrupted stability. To date, only manually controlled impulsive take-off approaches have been demonstrated in the literature.

\section{Gradual transition modeling and control}

Gradual transitions can be further classified into horizontal and vertical. Horizontal gradual transitions are typically used by vehicles that are able to float on the water surface, and make use of conventional UAV propulsion systems to accelerate to take-off speed [12], [21]. During this process, the UAAV transitions from a slow dragging regime where wave drag is dominant and buoyancy supports most of its weight to a fast planing regime where friction drag is dominant, and its weight is supported by hydrodynamic lift. Fuselages have to be carefully designed to achieve this behaviour, and whilst the literature on seaplane hull design is extensive [31] not all guidelines are relevant at the MAV scale. As mentioned in [12], ground effect has a significant impact on take-off at this scale. On one hand, it provides considerably more lift, but on the other hand, it hastens the onset of stall. The water surface state also plays a key role, small chop being especially detrimental in the ability of such UAAVs to take off. Due to the above factors, the take-off of small-scale UAAVs can in fact be challenging to automate, and to the authors' knowledge autonomous horizontal take-off of small-scale UAAVs has not been demonstrated.

Another type of gradual transition is VTOL, which is not as affected by the water surface state. Standard multirotors which are adapted for water interaction, such as [9], [14], [23] require little adaptation to perform this. However, it is more complex to achieve this on fixed-wing designs. As discussed in [16], water interaction needs to be accounted for, and more importantly, gyroscopic effects originating from the propulsion system's torque and spin induce an undesirable yaw rate. Only a small number of studies have considered VTOL manoeuvres for aerial-aquatic vehicles. Moore [2] demonstrates a VTOL-like approach for a delta-wing vehicle transitioning from a floating state to flight, based on predefined smooth exit trajectories and a feedback control law. An alternative approach is presented in Tétreault et al. [17], who suggest a two-phase active control strategy for a fixed-wing UAAV. The vehicle initially main- 


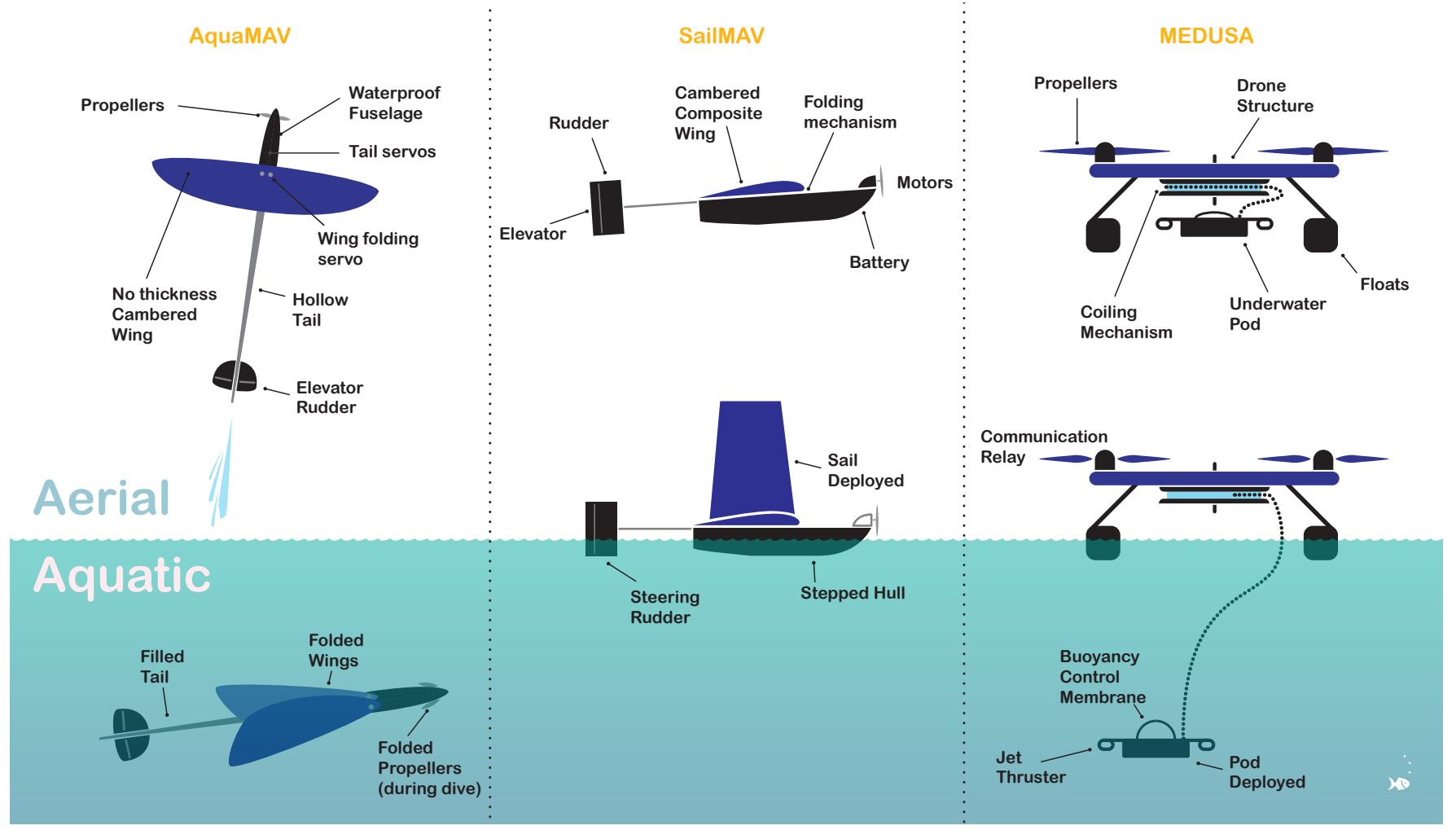

Figure 2: (left to right) Sideview of the AquaMAV, SailMAV and MEDUSA both in their aerial and aquatic configuration. The structure, actuation and adaptation thereof is indicated with arrows.

tains a vertical trajectory until it has gained some altitude and speed, then transitions to level flight. Both phases are handled by the same PD control law, albeit with different gains and references. Whilst the first strategy is more effective, it relies on pre-computed trajectories which can be model reliant. Even though less effective, the second approach is simpler to implement and is less model-reliant.

\section{EXAMPLES OF HYBRID LOCOMOTION MODES}

Hybrid locomotion can be a versatile tool in designing unusual mission profiles. However, there are rarely ready-made solutions that can easily be implemented in UAAVs. While general design principles may be applicable to different robots, especially ones within similar categories, detailed development of effective UAAVs needs to be carefully adapted to each specific mission. As shown in Sec. II, UAAVs are generally designed with different mission profiles in mind, and thus require widely different actuators, lifting surfaces and propulsion units. In this section we discuss three specific but representative UAAVs, which are shown in Fig. 2. These are designed for three different types of missions and illustrating a number of general challenges and potential solutions in UAAV design.

\section{A. Long-duration water surface sampling}

Long duration missions in calm bodies of water are attractive to study spatial and temporal scales in remote water-bodies. The SailMAV [12] can perform long-duration missions by harvesting wind energy as a form of propulsion. As shown in Fig 2, sections of its wing can pivot along a longitudinal axis to a vertical position. A second Degree of Freedom (DoF) is added to each wing to allow its orientation to change as required by the wind, a feature also used in flight for roll control. A custom actuator with two positions locking is required to alternate the wings between flight and sail mode. The system, thus, self-locks at the wing position (dihedral angle of $6^{\circ}$ ) and at the sail position (dihedral angle of $90^{\circ}$ ), using power only during the morphing process. Actuation of morphing surfaces should not be non-backdrivable to conserve power, like worm-gear mechanisms which have a high transmission ratio, slowing down the actuation, or clutch bearings and pawl-gears which are only one-way drivable, which can be limiting for some robots.

The control needed for autonomous take-off and landing of seaplanes is still a challenge due to the complexity of hydrodynamic forces and the chaotic perturbation induced by the sea-state. Methods less dependent on wave measurements (slope angle and height) should be developed for a feasible implementation on UAAVs (as opposed to [32], [33]).

\section{B. Impulsive single point sampling}

Plunge diving UAAVs, such as the AquaMAV [13], can perform single point sampling in small or cluttered water-bodies. These sustain high impact loads in transition, thus some methods to reduce the wing's aspect ratio is usually advantageous. Folding the wings, similar to what a plunge-diving gannet would do, reduces the frontal area of the UAAV, drastically reducing loading. Due to the reduced control during this phase, the wing sweeping needs to be as accurate as possible to avoid unwanted roll or yaw during the dive. Continuous wing sweep positions could improve the level of control obtained during the dive, but is still not demonstrated in the literature. It is generally advantageous to use a single propulsion system 
for the aerial and aquatic phase. However, the large difference in Reynolds number between water and air, means that the optimal operation point for any given motor-propeller combination is also largely different. Impulsive transition to air is the defining characteristic of these platforms. It provides the large impulse required for take-off from underwater, which would not be possible with a propulsion system developed for flight. This has previously been achieved using compressed $\mathrm{CO}_{2}$.

The control for both plunge-diving and impulsive take-off is quite challenging due to the small amount of time allowed to do those transitions. During the preparation stage, the UAAV should be in a suitable state in order to reduce the control required during the actual transition phase, taking into account disturbances to make sure that the state is valid before engaging the transition. In addition, the control has to take into account the actuation speed of the mechanical components.

\section{Maneuverable underwater long-duration mission}

As discussed above, although shared actuation is highly desirable in hybrid locomotion, it also incurs complexity in UAAV design. MEDUSA [14] explores the usage of a dual system, taking advantage of an off-the-shelf multirotor modified to float on the water, and a tethered submarine pod. The pod can control its depth, forward motion and heading in the horizontal plane, which are the 3 DoFs required to explore underwater environments.

The pod inherits design features from AUVs, however it also takes advantage of its connection to the quadcopter at the surface. The horizontal movement is handled by differential water-jet thrusters on each side of the pod, allowing the pod to move freely in the horizontal plane [34]. Depth control in AUVs is usually done by having the drone neutrally buoyant and using the propellers to thrust up/down in the water [23], or by changing its buoyancy by taking water into compartments as needed. In this case however, there is permanent access to atmospheric air, so the volume of the underwater pod can be changed using a micro-air-pump. This is achieved by inflating a soft membrane forming a dome, changing the volume and therefore the buoyancy of the pod.

The underwater control and navigation are topics well covered for purely underwater AUVs, where actuators are chosen and arranged to simplify the control. For UAAVs where weight is a constraint, having a limited number of actuators greatly limits the feasible control and actuation.

\section{DisCUSSION}

Aerial-aquatic MAVs is a recent field of research. While successful prototypes have been demonstrated in favorable conditions, there are still significant obstacles to practical adoption of this technology. In this section, we reiterate some of the current control-related challenges in the field and highlight opportunities for future work.

A crucial limitation in the majority of existing UAAV research is the lack of consideration of environmental disturbances and realistic operating conditions. For small-scale UAAVs, water surface forces are disturbance forces that cannot be neglected when designing a control system. This raises the need to extend the research on wave performance and robustness (mainly studied for larger ships) to small scale vessels. Two axes should be explored in this effort, namely, physical intelligence and disturbance rejection. The former involves making the drone inherently robust by design, while the latter hinges on devising robust control schemes that are suitable for lightweight UAAVs. The latter is crucial for transition methods that are affected by the sea state. In this context, an effective estimator of the surface state is essential, however, only methods that hinge on costly optimisation schemes have been demonstrated so far.

Autonomous and robust control of underwater-to-air transitions, especially for fixed-wing configurations, is still relatively lacking, with many new designs showing only manually controlled transitions. There is a scope for exploring more reliable, stable and efficient transition manoeuvres that will take the developed robots closer to autonomous operation capability. Underwater manoeuvrability and efficiency are also highly limited in many robots, which constrains the types of missions they can accomplish. Particularly operating in the vicinity of critical infrastructure or fragile ecosystems will require a high degree of agility and precision that has yet to be demonstrated in existing UAAVs.

A continued growth of the multi-modal robot field will also require reliable frameworks for control and autonomy. This entails both developing conceptual control schemes that can be easily adapted to different types of UAAVs, and integrating these in existing software suites for robots. While numerous autopilots are available for standard drones (PX4, ArduPilot, Betaflight), they have only limited multi-modal capabilities, particularly in terms of transitions between locomotion modes. Custom modifications have to be implemented for UAAVs, especially for the sub-aquatic mode, where robots lose their radio and GPS link; thus needing some level of autonomy.

High levels of autonomy will rely on underwater-compatible sensors, which usually come at a large payload cost. However, pose and localisation, which are essential for autonomy, should be measured by a small set of sensors. While pose estimates can be obtained from regular autopilots, localisation is a known issue for underwater robots due to unavailability of GPS, low range of communication and poor lighting conditions. Here, an interesting prospect for UAAVs is the usage of surface buoys relaying position using acoustic wave communication [35].

Water surface locomotion involves additional challenges of its own. An essential requirement for sailing UAAVs, for example, is the ability to sense wind direction. While previous research has used a miniaturised wind arrow whose orientation is read by a magnetic encoder, a perhaps more attractive solution is the usage of ultrasound anemometers, which are typically waterproof by design and have no moving parts. However, the currently available instruments are too heavy for the typical scale of UAAVs.

In general, the development of suitable instrumentation at small scales is one of the key challenges in UAAV research, and further efforts are required in this domain. 


\section{CONCLUSION}

This short paper reviewed the challenges and requirements involved in autonomous operation and control of small-scale unmanned aerial-aquatic vehicles (UAAVs). A brief but comprehensive highlevel overview of the relevant literature was given, with emphasis on control, actuation and sensing. Categorising existing UAAV designs according to their key features, allowed for the different requirements arising from each design type to be elucidated, and for general observations to be made. Specific robots were used as examples to illustrate the main points more clearly and provide the reader with useful practical insight. The paper also puts forth recommendations on how the main challenges in the field can be addressed and identifies promising areas of research, thus paving the way for new research in UAAV autonomy and control.

\section{REFERENCES}

[1] R. Zufferey, S. Armanini, A. Farinha, and M. Kovac, "Adaptive Morphology in Aerial-Aquatic Robots," 9th International Symposium on Adaptive Motion of Animals and Machines (AMAM 2019), 2019, publisher: EPFL, Lausanne, Switzerland. doi: 10.5075/EPFL-BIOROB-AMAM2019-38.

[2] J. Moore, A. Fein, and W. Setzler, "Design and analysis of a fixed-wing unmanned aerial-aquatic vehicle," in 2018 IEEE International Conference on Robotics and Automation (ICRA), Brisbane, QLD, Australia, 2017.

[3] S. Sareh, R. Siddall, T. Alhinai, and M. Kovac, "Bio-inspired soft aerial robots: adaptive morphology for high-performance flight," in Soft Robotics: Trends, Applications and Challenges. Cham: Springer International Publishing, 2017, pp. 65-74.

[4] R. Siddall and M. Kovač, "Launching the AquaMAV: Bioinspired design for aerial-aquatic robotic platforms," Bioinspiration and Biomimetics, vol. 9, no. 3, 2014, doi: 10.1088/1748-3182/9/3/031001.

[5] A. Narayanan, P. Rajeshirke, A. Sharma, and K. Pestonjamasp, "Survey of the emerging bio-inspired Unmanned Aerial Underwater Vehicles," IOP Conference Series: Materials Science and Engineering, vol. 810, no. 1, p. 012078 , Mar. 2020, publisher: IOP Publishing. doi: 10.1088/1757-899X/810/1/012078.

[6] S. Qiu and C. Weicheng, "An Overview on Aquatic Unmanned Aerial Vehicles," Annals of Reviews and Research, vol. 5, no. 3, pp. 10-28, Nov. 2019, doi: 10.19080/ARR.2019.05.555663.

[7] R. Siddall and M. Kovac, "Fast Aquatic Escape with a Jet Thruster," IEEE/ASME Transactions on Mechatronics, vol. 22, no. 1, pp. 217-226, 2017, doi: 10.1109/TMECH.2016.2623278.

[8] W. Stewart, W. Weisler, M. MacLeod, T. Powers, A. Defreitas, R. Gritter, M. Anderson, K. Peters, A. Gopalarathnam, and M. Bryant, "Design and demonstration of a seabird-inspired fixed-wing hybrid UAV-UUV system,' Bioinspiration \& Biomimetics, vol. 13, no. 5, p. 056013, Aug. 2018, doi: 10.1088/1748-3190/aad48b.

[9] H. Alzu'bi, I. Mansour, and O. Rawashdeh, "Loon Copter: Implementation of a hybrid unmanned aquatic-aerial quadcopter with active buoyancy control,' Journal of Field Robotics, vol. 35, no. 5, pp. 764-778, Aug. 2018, doi: 10.1002/rob.21777.

[10] M. Maia, P. Soni, and F. Diez-Garias, "Demonstration of an aerial and submersible vehicle capable of flight and underwater navigation with seamless air-water transition," (arXiv:1507.01932), 2015.

[11] D. Lu, C. Xiong, H. Zhou, C. Lyu, R. Hu, C. Yu, Z. Zeng, and L. Lian, "Design, fabrication, and characterization of a multimodal hybrid aerial underwater vehicle," Ocean Engineering, vol. 219, p. 108324, 2021, doi: 10.1016/j.oceaneng.2020.108324.

[12] R. Zufferey, A. Ortega, C. Raposo, S. F. Armanini, A. Farinha, R. Siddall, I. Berasaluce, H. Zhu, and M. Kovac, "Sailmav: design and implementation of a novel multi-modal flying sailing robot," Robotics and Automation Letters, vol. 4, no. 3, 2019, doi: 10.1109/LRA.2019.2921507.

[13] R. Siddall, A. Ancel, and M. Kovač, "Wind and water tunnel testing of a morphing aquatic micro air vehicle," Royal Society Interface Focus, vol. 7, no. 1, p. $20160085,2017$.

[14] D. Debruyn, R. Zufferey, S. F. Armanini, C. Winston, A. Farinha, Y. Jin, and M. Kovac, "Medusa: a multi-environment dual-robot for underwater sample acquisition," IEEE Robotics and Automation Letters, vol. 3, no. 4, 2020.
[15] R. Zufferey, A. Ortega, A. Farinha, R. Siddall, S. F. Armanini, M. Nasr, R. Brahmal, G. Kennedy, and M. Kovac, "Consecutive aquatic jump-gliding with water-reactive fuel," Science Robotics, vol. 4, no. 34, 2019.

[16] R. Peloquin, D. Thibault, and A. L. Desbiens, "Design of a passive vertical takeoff and landing aquatic UAV," IEEE Robotics and Automation Letters, vol. 2, no. 2, pp. 381-388, April 2017.

[17] E. Tétreault, D. Rancourt, and A. L. Desbiens, "Active vertical takeoff of an aquatic UAV," IEEE Robotics and Automation Letters, vol. 5, no. 3, pp. 4844-4851, 2020.

[18] G. Pisanich and S. Morris, "Fielding an amphibious UAV: development, results, and lessons learned," in Proceedings. The 21st Digital Avionics Systems Conference, vol. 2, Irvine, CA, USA, 2002, pp. 8C4-8C4.

[19] G. Meadows, E. Atkins, P. Washabaugh, L. Meadows, L. Bernal, B. Gilchrist, D. Smith, H. VanSumeren, D. Macy, R. Eubank, B. Smith, and J. Brown, "The flying fish persistent ocean surveillance platform," in AIAA Infotech@Aerospace Conference, ser. Infotech@Aerospace Conferences. Seattle, Washington, USA: American Institute of Aeronautics and Astronautics, 2009, doi: 10.2514/6.2009-1902.

[20] X. Yang, T. Wang, J. Liang, G. Yao, and M. Liu, "Survey on the novel hybrid aquatic-aerial amphibious aircraft: Aquatic unmanned aerial vehicle (AquaUAV)," Progress in Aerospace Sciences, vol. 74, pp. 131-151, 2015 doi: 10.1016/j.paerosci.2014.12.005

[21] G. Yao, J. Liang, T. Wang, X. Yang, M. Liu, and Y. Zhang, "Submersible unmanned flying boat: Design and experiment," in 2014 IEEE International Conference on Robotics and Biomimetics (ROBIO 2014), 2014, pp. 1308-1313.

[22] A. Gao and A. H. Techet, "Design considerations for a robotic flying fish," in OCEANS'11 MTS/IEEE KONA, 2011, pp. 1-8, ISSN: 0197-7385.

[23] P. L. J. Drews, A. A. Neto, and M. F. M. Campos, "Hybrid unmanned aerial underwater vehicle: Modeling and simulation," in 2014 IEEE/RSJ International Conference on Intelligent Robots and Systems, Chicago, IL, USA, 2014, pp. 4637-4642.

[24] L. Ristroph and S. Childress, "Stable hovering of a jellyfish-like flying machine," Journal of The Royal Society Interface, vol. 11, no. 92, p. 20130992, 2014, publisher: Royal Society. doi: 10.1098/rsif.2013.0992.

[25] Y. Chen, H. Wang, E. F. Helbling, N. T. Jafferis, R. Zufferey, A. Ong, K. Ma, N. Gravish, P. Chirarattananon, M. Kovac, and R. J. Wood, "A biologically inspired, flapping-wing, hybrid aerial-aquatic microrobot," Science Robotics, vol. 2, no. 11, p. eaao5619, 2017, doi: 10.1126/scirobotics.aao5619.

[26] Y. H. Tan, R. Siddall, and M. Kovac, "Efficient aerial-aquatic locomotion with a single propulsion system," IEEE Robotics \& Automation Letters, vol. 2 no. 3, 2017.

[27] M. Zongcheng, C. Danqiang, L. Guoshuai, and Z. Junjie, "Constrained adaptive backstepping take-off control for a morphing hybrid aerial underwater vehicle," Ocean Engineering, vol. 213, p. 107666, 2020.

[28] Y. Chen, E. F. Helbling, N. Gravish, K. Ma, and R. J. Wood, "Hybrid aerial and aquatic locomotion in an at-scale robotic insect," in Intelligent Robots and Systems (IROS), 2015 IEEE/RSJ International Conference on. IEEE 2015, pp. 331-338.

[29] W. Stewart, W. Weisler, M. Anderson, M. Bryant, and K. Peters, "Dynamic modeling of passively draining structures for aerial-aquatic unmanned vehicles," IEEE Journal of Oceanic Engineering, vol. 45, no. 3, pp. 840-850, 2020

[30] S. F. Armanini, R. Siddall, and M. Kovac, "Modelling and simulation of a bioinspired aquatic micro aerial vehicle," in AIAA Aviation 2019 Forum, Dallas, Texas, 2019, p. 3115.

[31] I. Dathe and M. de Leo, "Hydrodynamic characteristics of seaplanes as affected by hull shape parameters," in Advanced Marine Vehicles Conference, Arlington, VA, U.S.A., 1989, doi: 10.2514/6.1989-1540.

[32] H. Du, G. Fan, and J. Yi, "Autonomous takeoff for unmanned seaplanes via fuzzy identification and generalized predictive control," in 2013 IEEE International Conference on Robotics and Biomimetics (ROBIO), Dec. 2013 pp. 2094-2099.

[33] D. Huan, F. Guoliang, and Y. Jianqiang, "Autonomous landing for unmanned seaplanes based on active disturbance rejection control," in 2015 34th Chinese Control Conference (CCC), Jul. 2015, pp. 5663-5668, iSSN: 1934-1768.

[34] S. A. Watson and P. N. Green, "Propulsion systems for micro-autonomous underwater vehicles ( $\mu$ auvs)," in 2010 IEEE Conference on Robotics, Automation and Mechatronics, 2010, pp. 435-440.

[35] X. Su, I. Ullah, X. Liu, and D. Choi, "A Review of Underwater Localization Techniques, Algorithms, and Challenges,” Journal of Sensors, vol. 2020, p. e6403161, Jan. 2020, publisher: Hindawi. doi: 10.1155/2020/6403161. 